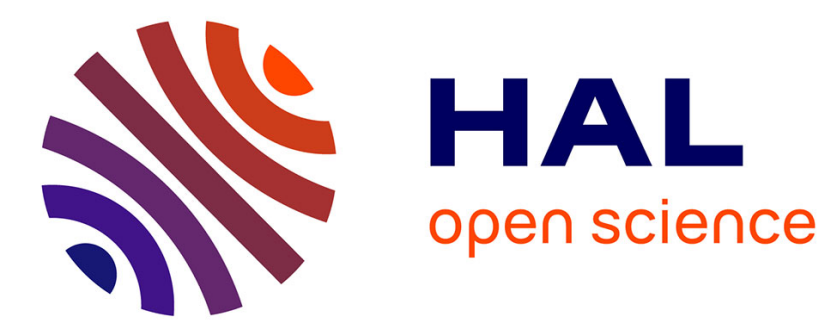

\title{
Numerical and experimental approaches to characterize the mass transfer process in wood elements
}

Tuan Anh Nguyen, Nicolas Angellier, Sabine Caré, Laurent Ulmet, Frédéric

Dubois

\section{- To cite this version:}

Tuan Anh Nguyen, Nicolas Angellier, Sabine Caré, Laurent Ulmet, Frédéric Dubois. Numerical and experimental approaches to characterize the mass transfer process in wood elements. Wood Science and Technology, 2017, 51 (4), pp.811 - 830. 10.1007/s00226-017-0898-5 . hal-01711379

HAL Id: hal-01711379

https://hal-enpc.archives-ouvertes.fr/hal-01711379

Submitted on 30 Apr 2019

HAL is a multi-disciplinary open access archive for the deposit and dissemination of scientific research documents, whether they are published or not. The documents may come from teaching and research institutions in France or abroad, or from public or private research centers.
L'archive ouverte pluridisciplinaire HAL, est destinée au dépôt et à la diffusion de documents scientifiques de niveau recherche, publiés ou non, émanant des établissements d'enseignement et de recherche français ou étrangers, des laboratoires publics ou privés. 
3 Tuan Anh N'Guyen ${ }^{(1)}$, Nicolas Angellier ${ }^{(1)}$, Sabine Caré( ${ }^{(2)}$, Laurent Ulmet ${ }^{(1)}$, 4 Frédéric Dubois ${ }^{(1)}$

6 Egletons, France

7 (2) Laboratoire Navier, UMR8205, Ecole des Ponts, IFSTTAR, CNRS, UPE, Champs-sur-

8 Marne, France

9 Corresponding author: Nicolas Angellier, Campus Universitaire de Génie Civil, 1017 Boulevard Jacques Derche, 19300 Egletons

11 Phone: (+33) 555934 520, Fax: (+33) 555934 501, e-mail: nicolas.angellier@ unilim.fr

\section{the Mass Transfer Process in Wood Elements}

Group (EA 3178), University of Limoges, F-19300

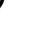


exchanges and equilibrium moisture. Scale effects and boundary conditions are also

27 investigated herein.

28 Keywords: Moisture content, Wood material, Mass transfer, Gammadensimetry,

29 Diffusion model, Inverse methods.

\section{1 - Introduction}

32 In pursuit of sustainable development goals, greater numbers of timber elements are being used in many types of public construction like buildings and bridges. The demand for

34 timber construction today is constantly rising, yet many technological obstacles still

35 prevent the wider use of wood in the highly competitive field of construction materials.

36 When placed in outdoor conditions or heat spaces, these elements are subjected to

37 moisture content variations induced by a non-stabilized equilibrium between wood and

38 the climatic environment. The moisture content gradient, associated with the orthotropy

39 of wood, induces hydric stresses amplified by the hyperstaticity level or the coupling with

40 other materials, such as concrete or steel in the case of composite structures. The

41 magnitude of these stresses is not well known at the design stage and not seriously taken

42 into account in construction regulations. This mechanical state can induce a degradation

43 in toughness and the appearance of cracks. In order to facilitate timber structure

44 development, the issue of moisture content needs to be addressed by the scientific

45 community.

46 This context requires in particular developing an in situ monitoring protocol. The control

47 of moisture content mapping through a cross-section is currently limited by resistive

48 methods that rely on the use of surface electrodes, which allow for the estimation, after

49 calibration, of moisture to a depth of around $1 \mathrm{~cm}$ (Dubois et al., 2006). This inadequate 
method needs to be complemented by numerical simulations in order to deduce moisture

51 content gradients and their evolution vs. time by taking into account temperature and humidity in the climatic environment (Manfoumbi, 2012; Manfoumbi, 2014). This approach however imposes several limitations. First of all, the diffusion properties introduced into numerical simulations must be correctly estimated by determining the nonlinear diffusion coefficients. Secondly, the simulation must integrate surface exchanges by considering the convective effects in terms of temperature and humidity. As a final step, specific thermo-hygro-mechanical behavior must be considered when estimating the thermo-hygro stresses (Dubois et al., 2012; Colmars et al., 2014).

The present work pertains to the first step and is intended to determine the diffusion parameters. The characterization protocol is typically based on the double weighing principle. For various sample geometries (at the centimeter scale), this technique consists of optimizing the diffusion parameters in accordance to their corresponding average moisture content during adsorption and desorption tests. The main difficulty lies in separating the effects of boundary conditions, including sorption hysteresis and surface humidity exchanges. Numerical algorithms, derived from a minimization step between experimental and numerical differences, do not always yield a unique realistic solution.

67 Moreover, in the case of samples far from the equilibrium state, errors on the identifications of the diffusion properties may be done due to insufficient measurement

69 points. In this case, the method doesn't allow the uncoupling between the diffusion to the

70 heart and the surface exchanges between first fibers and the climatic environment.

71 To overcome this inconsistency and to highlight how the choice of the experimental 72 measurements may influence the identification of the diffusion parameters, the present 73 paper provides an additional experimental test on larger samples (at the decimeter scale), 74 thus making it possible to generate a moisture content profile measured with the 
gammadensimetry technique. Three boundary condition tests have been taken into

76 account in order to demonstrate the relevance of this method and its robustness. The first

77 section will describe the experimental set-up through the selected sample geometries and

78 boundary conditions. This section will be completed by a presentation of the

79 gammadensimetry technique and its specific application in water profile measurements

80 during wetting or drying phases.

81 The numerical model and the determination of the diffusion parameters will be discussed

82 in a second section. A nonlinear Fick's law will be coupled with an inverse method based

83 on the downhill simplex method, which in turn allows optimizing the diffusion

84 parameters in accordance with average moisture content measurements and water profiles

85 determined by gammadensimetry method.

86 The last section will focus on experimental results. The method robustness will be

87 validated by exhibiting the effects of boundary conditions and geometric dimensions.

2 - Materials and methods: experimental set-up and numerical model

$89 \quad 2.1$ Materials and hydric loading

90 The primary target of the experimental tests was to monitor moisture content evolution

91 vs. time through various boundary conditions and geometries, using large samples at high

92 relative humidity increments. As shown in Fig 1, three configurations were proposed

93 considering moisture diffusion along the longitudinal direction. More precisely, the

94 samples had been cut out along the anisotropic directions (i.e. Longitudinal L along the

95 tree stem, Radial R perpendicular to the rings, and Tangential T parallel to the rings), with

96 moisture diffusing along the longitudinal direction. 


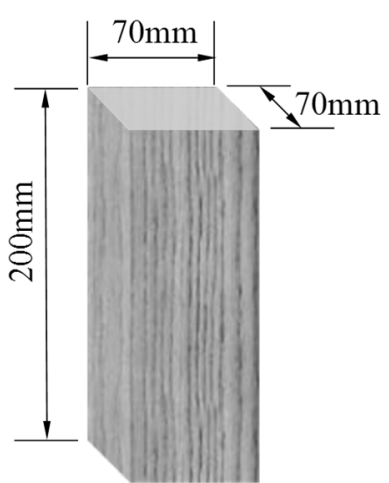

Sample (a)

97

\begin{tabular}{|c|c|c|c|}
\hline 0.6 & 5.1 & 9.5 & $\begin{array}{c}\text { Initial moisture } \\
\text { content (\%) }\end{array}$ \\
\hline 97 & 97 & 86 & $\begin{array}{c}\text { Relative Humidity } \\
(\% \mathrm{RH})\end{array}$ \\
\hline
\end{tabular}

Fig. 1 Moisture boundary conditions and sample geometries for the three cases

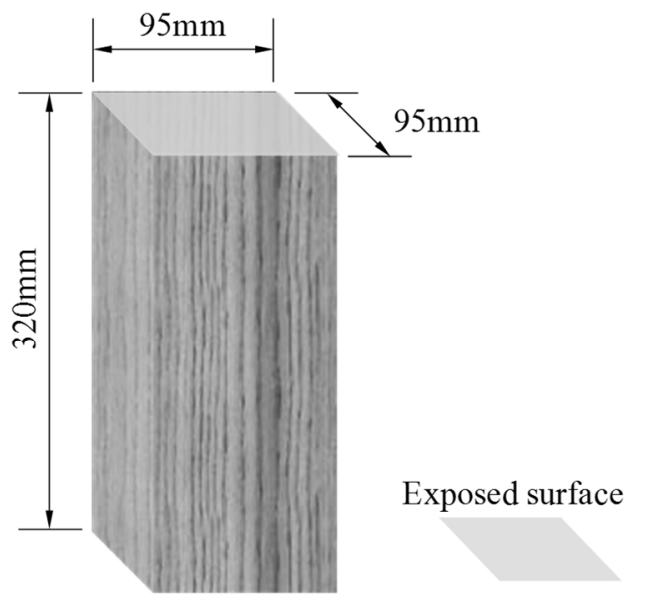

Sample (c)

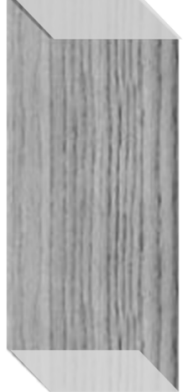

Sample (b) posed surface

. 
110 For one thing, all specimens were conditioned in a dry environment using several 111 equilibrium moisture contents for $w$ : less than $1 \%$ for sample (a), around $5 \%$ for sample

112 (b), and 9\% for sample (c). The moisture content $w$ is typically defined as the ratio of the 113 water mass ${ }^{M_{H_{2}} \mathrm{O}}$ to the dried or solid mass of wood ${ }^{M}$. Under a constant temperature 114 of approx. $19^{\circ} \mathrm{C}\left(+/-1^{\circ} \mathrm{C}\right)$, samples were placed in desiccators over saturated salt

115 solutions, which served to impose a constant relative humidity of around $97 \% \mathrm{RH}$ for 116 samples (a) and (b), and roughly $86 \% \mathrm{RH}$ for sample (c). Average moisture content is

117 basically determined by measuring weight during the sorption test.

\section{$118 \quad 2.2$ - Gammadensimetry method}

119 Gammadensimetry is a non-destructive method used to determine the density or moisture 120 content of civil engineering materials (Da Rocha et al., 2001; Villain and Thierry, 2006; 121 Ferraz and Aguiar, 1985). This technique is based on the absorption of gamma rays 122 emitted by a radioactive source (in our case Cesium $\mathrm{Cs}^{137}$ ) and follows Lambert's law. To 123 determine moisture content at various heights, it is assumed that the wood material is 124 composed of three phases, namely solid $s$ (dried wood), water $w$ (bound water) and air $a$, 125 such that:

$$
\ln \left(\frac{N_{o}}{N}\right)=\mu_{s} \cdot \rho_{s} \cdot x_{s}+\mu_{w} \cdot \rho_{w} \cdot x_{w}+\mu_{a} \cdot \rho_{a} \cdot x_{a}
$$

127 where $N_{O}$ is the number of incidental photons in the air, $N$ the number of photons

128 crossing the sample of thickness $X(\mathrm{~m})$, and $\mu_{i}, \rho_{i}$ and $x_{i}$ the mass absorption

129 coefficients $\left(\mathrm{m}^{2} / \mathrm{kg}\right)$, densities $\left(\mathrm{kg} / \mathrm{m}^{3}\right)$ and thicknesses $(\mathrm{m})$ of phases $i(i \in\{s, w, a\})$, 130 respectively. Let's remark that the air phase density and its mass absorption coefficient 
can both be neglected. If the gamma ray crosses a thickness $X$, the following can be expressed:

$$
X=\sum_{i} x_{i}
$$

134 The moisture content $w(\mathrm{t})$ is typically defined such that:

$$
w=\frac{M_{H_{2} 0}}{M_{s}}=\frac{\rho_{w} \cdot x_{w}}{\rho_{s} \cdot x_{s}}
$$

136 According to expression (1), thicknesses $x_{s}$ and $x_{w}$ at experimental time $t$ are written as 137 follows:

$$
x_{w}(t)=\frac{\left(\ln \frac{N_{0}}{N}\right)_{t}-\left(\ln \frac{N_{0}}{N}\right)_{t=0}}{\mu_{w} \rho_{w}}+x_{w}(t=0)
$$

$$
x_{s}(t)=x_{s}(t=0)=\frac{\left(\ln \frac{N_{0}}{N}\right)_{t=0}}{\mu_{s} \rho_{s}+\mu_{e} \rho_{s} w_{(t=0)}}
$$

140 Due to swelling of the wood material under a sorption process, the water content may be

141 underestimated given that the thickness $x_{s}$ at time $t$ may be overestimated in the relation

142 (3). Nevertheless, the error on the moisture content due to the swelling effects in the

143 transverse plane (RT) is in the same magnitude order that the estimated swelling strains.

144 So, it can be neglected in regards to the dispersion of the measurements in link with the

145 further discussion about the possible uncertainties. In relation (5) therefore, it may be

146 assumed that thickness $x_{s}$ crossed by the gamma ray does not vary during the sorption

147 process to simplify the determination of the moisture content.

148 Moreover, the moisture content profile was assumed to be homogeneous in the sample at

149 time $t=0$. The calculus applied also had to integrate the Parafilm thickness in accordance

150 with expression (5). 
151 For the various phases, the densities were: $\rho_{w}=1,000 \mathrm{~kg} / \mathrm{m}^{3}$ and $\rho_{s}=1,520 \mathrm{~kg} / \mathrm{m}^{3}$. The mass absorption coefficient of each phase was calculated from its corresponding chemical

153 analysis, while the elementary mass absorption coefficients for the radiation energy of

$154 \mathrm{Cs}^{137}$ are given, for instance, in the tables published by the U.S. National Bureau of

155 Standards. For the water phase, the mass absorption coefficient was: $\mu_{w}=8.57 \cdot 10^{-3} \mathrm{~m}^{2} / \mathrm{kg}$.

156 As for the dried wood (solid) phase, it was assumed that wood is composed of cellulose,

157 hemicellulose and lignin, thus leading to a mass absorption coefficient $\mu_{s}=8.17 \cdot 10^{-3}$

$158 \mathrm{~m}^{2} / \mathrm{kg}$. More precisely, it was considered that the dried wood phase contained $50 \%$

159 carbon, $6 \%$ hydrogen and $44 \%$ oxygen in mass terms. Slight variations in these

160 percentages however do exert a small impact on the final mass absorption coefficient

161 value.

162 As shown in Fig 2, the moisture content profiles required placing the specimen in a plate

163 that was vertically moved by a robot through the gamma ray beam at a 5-mm step.

164 According to diffusion kinetics, it was assumed that moisture content is homogeneous

165 along the radial direction for each cross-section and solely dependent on the longitudinal

166 position of the gamma ray. Under these conditions, for each gamma ray position, the

167 average moisture content in the cross-section was measured. Regular profile

168 measurements were conducted throughout the experimental period ( 2 to 10 months).

169 Several profiles were measured (2 for samples (a) and (b), 3 for sample (c)), yet only the

170 averaged profile will be considered for the purpose of identifying diffusion parameters.

171 The viability of the gamma-ray method has been shown by Ferraz and Aguiar (Ferraz and

172 Aguiar, 1985) for determining density and moisture content of wood samples. Relative

173 deviations (absolute errors) between the traditional gravimetric method and the gamma-

174 ray method have been estimated. The differences between the two methods are estimated

175 up to $3 \%$ for samples with moisture content MC between $9 \%$ and $30 \%$ (relative error 
176 inferior to $20 \%$ ). The exactness of the gamma-ray method is limited mainly by errors

177 made in the determinations of $\mathrm{N}$ and No as a consequence of random disintegration; the

178 errors made in the determination in the other parameters (eg. $\mathrm{x}_{\mathrm{s}}$ ) are negligible. Moreover,

179 in the case of the use of the Cesium $\mathrm{Cs}^{137}$ radioactive source, the sensitivity of the method

180 may be low because of the small differences between the mass absorption coefficients of

181 water and dried wood (solid) phase. But despite these possible absolute errors, it is

182 possible to obtain an average moisture content profile for large samples subjected to

183 hydric loading.



184

185 Fig 2 Gammadensimetry profiles A and B for samples (a) and (b) along the longitudinal direction (for sample (c), three profiles A, B and C have been considered)

188 Ultimately, the average moisture content profiles provided by gammadensimetry and 189 weighing must allow for the characterization of diffusion properties. The next sections

190 will propose an inverse method technique based on a mass transfer algorithm, along with 191 a downhill simplex method that serves to minimize the difference between numerical 192 model output and experimental measurements. This work will be limited to a uniaxial 193 approach due to moisture content homogeneity within the cross-sections. 


\section{3 - Diffusion model}

195 Many models are proposed or developed to describe diffusion in wood: Fickian models,

196 multi or not Fickian models, which can take into account dependence of diffusion

197 coefficient on moisture content and take into account the surface exchanges. In the

198 general case, the choice of the mass transport model deals with the necessity (or not) of

199 separation of bound liquid, free water and vapor (Krabbenhoft 2003; Perre and Turner,

200 2008). In the present work, we are not in drying case from high water content where

201 liquid, bound water and vapor cohabit, but below the saturation fibers point: in the

202 hygroscopic domain, only bound water and vapor have to be considered. In this case,

203 there is a common model with only one variable for both forms of water (Jakiela et al.,

204 2008; Rozas et al., 2009; Olek et al., 2011; Da Silva et al., 2011) or model with separated

205 variables (Frandsen et al., 2007, Krabbenhoft 2003, 2004). . Then, differences between

206 models can come from the choices for the treatment of the convective boundary

207 conditions: relaxation term (Olek et al., 2016), hydrous surface exchange coefficient. It

208 can be noticed that there is no consensus on the diffusion models which may have to be

209 used and on the convective boundary conditions. Our assumptions and geometries allow

210 us to use the common model which is described below.

211 The mass transfer process over time $t$ is typically modeled using Fick's second nonlinear

212 law. Fick's coefficients and the surface emission and diffusion coefficients are all

213 described as phenomenological parameters since they can only be grasped or

214 conceptualized through experience. According to isothermal conditions, the mass transfer

215 process integrates a relationship focused on non-linearity between moisture content level

216 and orthotropic diffusion tensor. From the perspective of a uniaxial representation

217 characterized by the $x$ coordinate, the time/space differential equation can be written as

218 follows (Perre and Degiovanni, 1990; Merakeb et al., 2006): 


$$
\frac{\partial w}{\partial t}=\frac{\partial}{\partial x}\left(D_{w}(w) \cdot \frac{\partial w}{\partial x}\right)
$$

220 The mass transfer process is principally driven by the diffusion property $D_{w}$. In wood materials, this property is admitted to be nonlinear with respect to moisture content, in accordance with the following form (Droin Josserand et al., 1989):

$$
D_{w}(w)=D_{o} \cdot \exp (k \cdot w)
$$

where $D_{0}$ is the anhydrous moisture diffusion coefficient, and $k$ a constant reflecting the nonlinear effect.

According to boundary conditions, solving Fick's equation requires knowledge of the surface moisture content, denoted $\partial \Omega$. So, if $S$ designates the hydrous surface exchange coefficient, then the diffusion equation can integrate the following boundary limits:

$$
D_{w}(w) \frac{\partial w}{\partial x}=S \cdot\left(w_{e}(t)-w_{\Omega}(t)\right)
$$

where $w_{\Omega}$ is the surface moisture content. To take into account the exchanges between water vapor molecules in air and bound water molecules in wood, it is commonly considered to employ a virtual exchange layer defined by an instantaneous equilibrium between external fibers and water vapor in the neighboring climatic environment. This equilibrium is usually driven by sorption hysteresis and defined by the virtual moisture content value, called $w_{e}$ (Lasserre, 2000; Merakeb et al., 2009).

236 Moreover, our problem can be reduced to characterizing $D_{o}, k, S$ and $w_{e}$. The mass 237 transfer subroutine is implemented as part of the finite element method. However, the 238 problem is further reduced to a uniaxial configuration, and expressions (6) through (8) 239 are solved using a finite difference method based on a time discretization based on an explicit Euler scheme (Zhou et al., 2011; Liu and Simpson, 1999). 


\section{4 - Downhill simplex method}

242 The downhill simplex method is introduced here to characterize diffusion properties

243 (Nedler, 1965). This method consists of minimizing the objective function in using only

244 function values and without calculating the derivative, so as to represent the gap between

245 the moisture content evolution during experimental time and model results from the

246 standpoint of least squares by means of adjusting diffusion parameters, such as diffusion

247 coefficient $D_{o}$, nonlinear parameter $k$, hydrous surface exchange $S$ and equilibrium

248 moisture content $w_{e}$. For these four unknowns, the method considers a geometric

249 polyhedron, called simplex, composed of 5 nodes, with each node representing a unique

250 solution. Based on a hierarchy of errors for each solution, this algorithm reduces the

251 geometric surface until convergence is reached. The vertices of this polyhedron will

252 undergo geometric transformations in moving towards a global minimum (Lagarias et al.,

253 1998). The simplex algorithm iterations correspond to simple algebraic operations on the

254 polygon vertices for elementary geometric transformations (reflection, contraction and expansion) (Kelley, 1999). Each iteration transformation solely depends on a series of

comparisons between objective function values corresponding to the calculated points and values of the polygon vertices to replace the worst vertex (maximum) by the new

258 fixed point. The polygon is thus reflected, extended and reduced depending on the 259 function shape until its optimum corresponds to a complete reduction of the simplex 260 yielding the optimal solution. As demonstrated by Kouchade (2004), this method is well

261 adapted to incomplete experimental curves.

262 The mass transfer process however performs two competing effects, in accordance with

263 expressions (6) and (8). Based solely on the average moisture content evolution, the 264 downhill simplex method does not distinguish the surface effect characterized by $S$ and $265 w_{e}$ from the mass diffusion characterized by $D_{o}$ and $k$. When limited to the average 
moisture content data, the minimization method can actually yield an infinite number of

267 solutions. The use of water profiles provided by gammadensimetry could ensure an acceptable level of separation between surface and mass effects.

\section{3 - Results and discussion: moisture content profiles of sorption tests and} identifications of diffusion parameters

271 This section proposes comparing the two methods for identifying diffusion parameters by taking into account both moisture loading and sample geometry. This methodology will be applied to samples (a) and (b) and then to sample (c). The diffusion parameters will be

274 discussed afterwards.

\section{1 - Identification of diffusion parameters for sample (a): reference case}

276

277

Sample (a) is characterized by just one surface of moisture content exchange, with initial drying in an oven at $103^{\circ} \mathrm{C}$. As shown in Fig. 3 after 81 days of adsorption, the average moisture content increased to only $14 \%$, i.e. far below the equilibrium moisture content estimated to lie around $22 \%$ at about $20^{\circ} \mathrm{C}$, according to Loulou (2013) and Merakeb et al. (2009). The equilibrium moisture content value is highly sensitive to both the moisture and thermal loading; hence, the level of precision is approx. $+/-2 \%$, especially for a high Relative Humidity loading.

Diffusion parameters are normally characterized using a simple weighing of small samples corresponding to average moisture content information over time; this procedure is referred to as standard identification. For this standard identification, the equilibrium state is normally raised, leading to a possible identification of the diffusion parameters. In our particular case, let's set the equilibrium moisture content value $w_{e}$ as unknown. In our example therefore, this approach is being compared on five weighing measurements (at 3, 10, 18, 25 and 81 days) with a so-called coupled identification using crossing 
weights and gamma-ray measurements.

291 The comparison between these two identification methods is presented in Fig. 3 and Fig.

292 4, in accordance with identified diffusion parameters (see Table 1). The nonzero values 293 obtained for the non-linearity coefficient and the rather low hydrous surface exchange

294 coefficient serve to validate the hypotheses of nonlinear diffusion and exchanges by 295 hydrous convection.

Tab 1 Diffusion parameters for sample (a)

\begin{tabular}{ccccc}
\hline Identification method & $D_{0}\left(m^{2} \cdot s^{-1}\right)$ & $k$ & $S\left(m \cdot s^{-1}\right)$ & $w_{e}$ \\
Standard identification & $4.50 \cdot 10^{-9}$ & 1.12 & $5.03 \cdot 10^{-8}$ & $20,6 \%$ \\
Coupled identification & $3.27 \cdot 10^{-9}$ & 1.94 & $4.73 \cdot 10^{-8}$ & $22,5 \%$ \\
\hline
\end{tabular}

298 Results highlight the sensitivity of identification by means of the inverse method. Fig. 3

299 illustrates that in terms of average moisture content evolution, various diffusion 300 parameters can yield the same results, especially within the experimental time frame.



Fig. 3 Results of standard and coupled identification in terms of average moisture content evolution for sample (a) 
305 The coupled identification was performed using five profiles (at each weighing time), but

306 only three profiles have been presented for the sake of clarity. The profile results

307 displayed in Fig. 4 however reveal slight differences. As explained previously, it can be

308 observed that the gammadensimetry measurements present dispersions around an average

309 profile value. Despite this dispersion (and some anomalous points), it is still possible to

310 compare the experimental and numerical profiles. Experimentally, we can observe that

311 the moisture content first increases on the exposed face and only in the first quarter of the

312 sample (day 3), then the moisture gradient decreases while the moisture content increases

313 up to the whole sample and the sealed face (day 81). Numerically, the main difference

314 between the profiles from the standard identification (that does not take into account the

315 experimental profiles) with those from the coupled identification concerns the higher

316 moisture content values on the exposed face as seen experimentally.

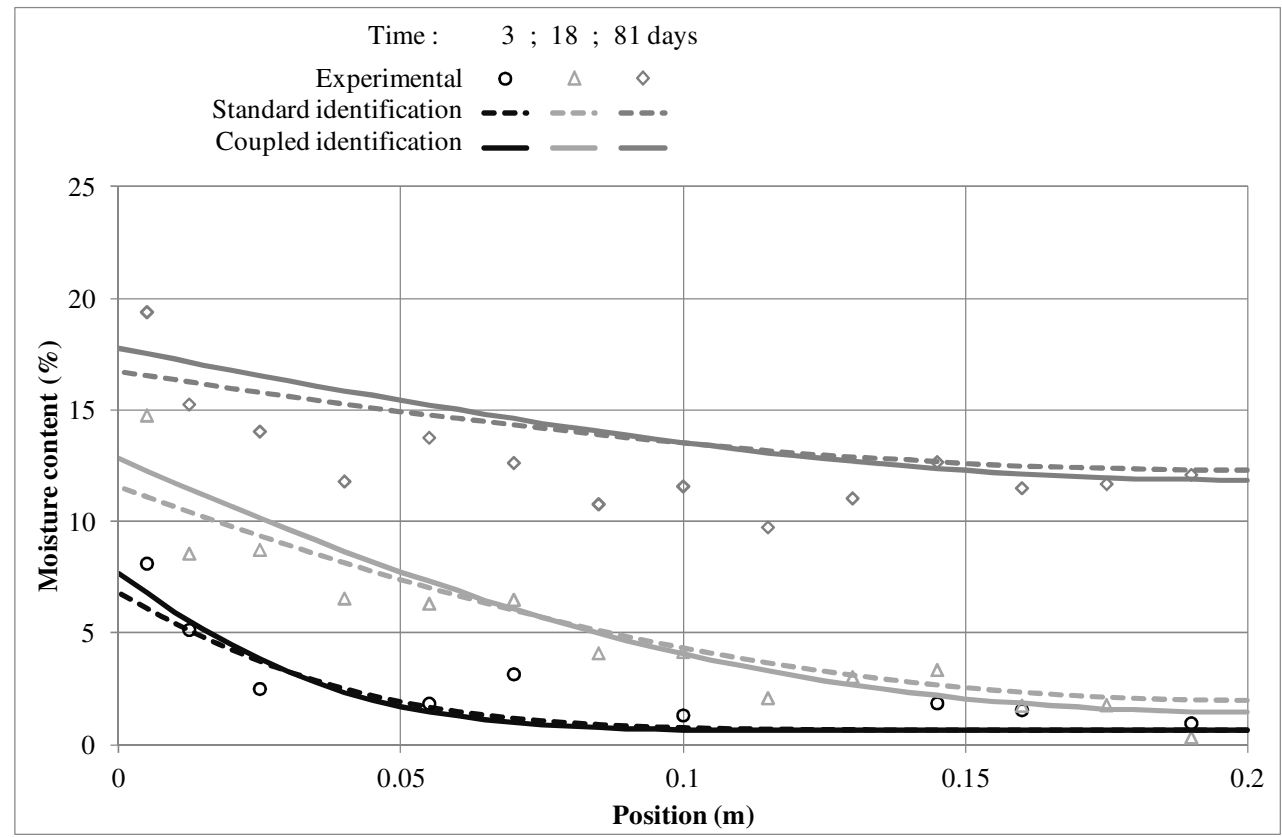

318 Fig. 4 Results of standard and coupled identification, in terms of moisture content 
320 As regards the identified diffusion parameters, let's note a slight difference in exchange

321 surface coefficient of around $6 \%$ and a difference in the anticipated value of $w_{e}(9 \%)$.

322 The main differences actually pertain to the diffusion parameter characterization and its

323 property of non-linearity. According to expression (7), the identification method produces

324 a great difference (of up to 25\%) in the diffusion parameter evolution vs. moisture content

325 (Fig. 5).

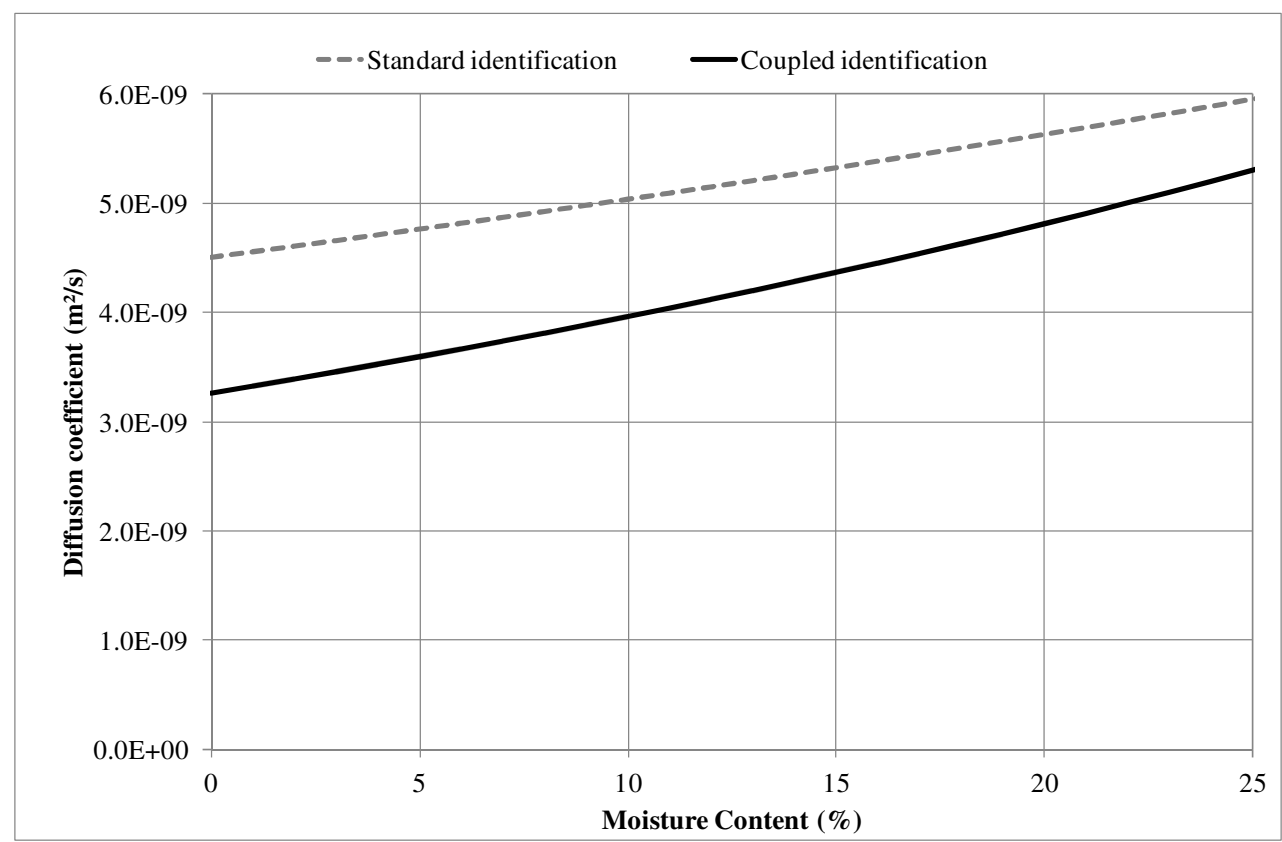

Fig. 5 Diffusion coefficient vs. moisture content within the hygroscopic domain given by the two identifications performed on the results of sample (a)

329 According to a homogeneity assumption on the sample however, parameters $D_{o}$ and $k$

330 should be intrinsic material properties, while the surface exchange coefficient is 331 somewhat intrinsic to the experiment and its exposure conditions. The small correction 332 due to the coupled identification on its value has a significant effect on the diffusion 333 parameters values and we can expect a better identification of this lasts. 

conditions

336 In the following discussion, both boundary and initial conditions will be the focus of an

337 investigation considering two surface exchanges and various initial moisture contents.

338 According to Figure 1, this second experiment will concentrate on sample (b). To

339 investigate the decoupling between the intrinsic diffusion coefficient ( $D_{o}$ and $k$ ) and the

340 surface exchange coefficient $S$, sample (b) is to be the same specimen as sample (a), yet

341 the initial conditions and exchange conditions have been modified. After the first

342 experiment, the sample is once again dried until a homogeneous moisture content of 5\%

343 is obtained and two faces are in contact with the environment. As shown in Fig. 6 after

344 less than 80 days of adsorption, the average moisture content has already risen to $19 \%$,

345 i.e. not far below the expected equilibrium moisture content.

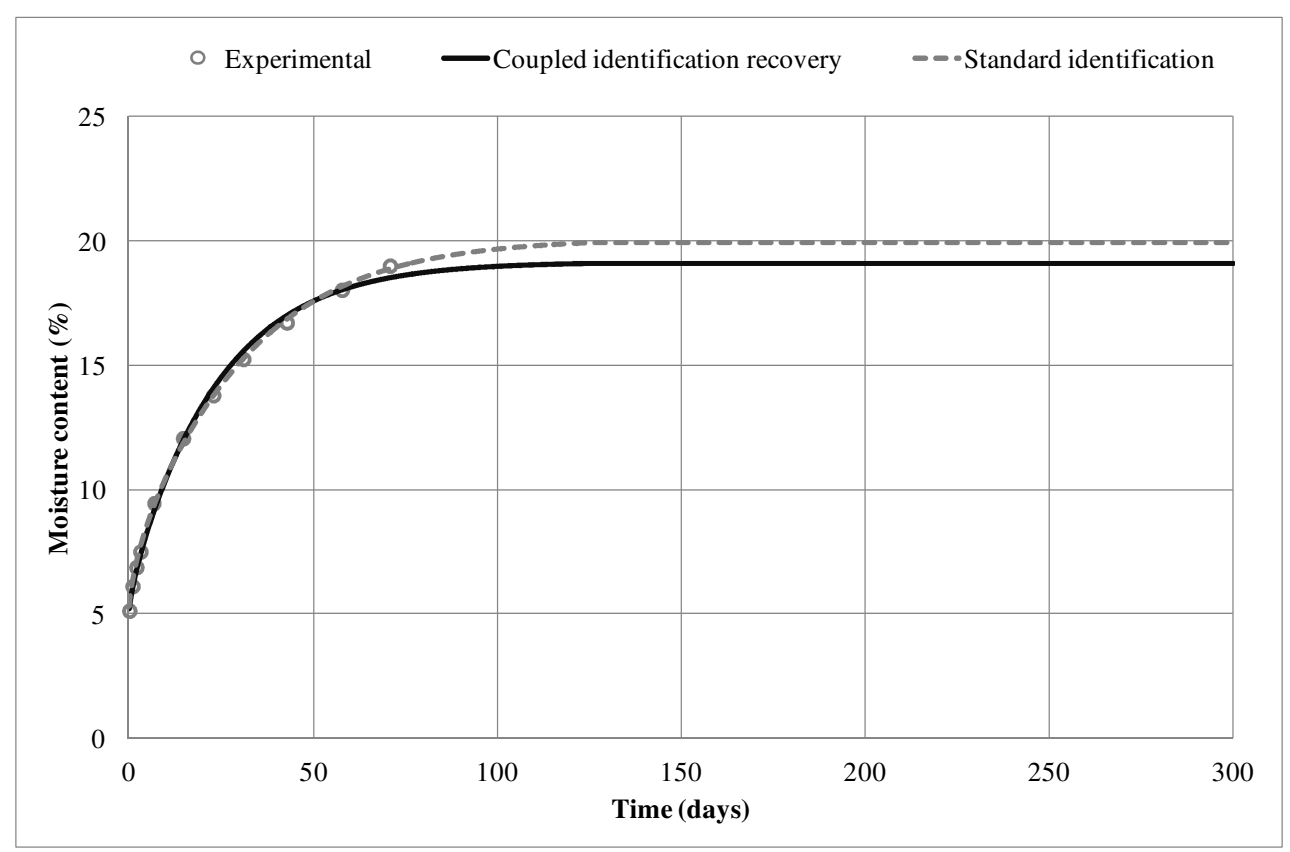

Fig. 6 Results from both the standard and coupled (recovery from sample (a))

349 These same approaches are then proposed in using diffusion coefficients from the coupled

350 identification of sample (a) in order to calibrate equilibrium moisture content and a 
351 second surface exchange coefficient $S^{\prime}$ that characterizes the additional surface

352 (assumption of a moisture content exchange asymmetry: surfaces and exposition being

353 the same, we can nevertheless not be sure that surface states, with wood rings, are so). It

354 can be noticed that the asymmetry may be not measured by the gamma-ray method owing

355 to moisture content dispersion. According to the diffusion properties listed in Table 2, the

356 average moisture content evolution and moisture content profiles can now be plotted

357 (Figs. 6 and 7).

Tab 2 Diffusion parameters for sample (b)

\begin{tabular}{cccccc}
\hline Identification method & $D_{o}\left(m^{2} \cdot s^{-1}\right)$ & $k$ & $S\left(m \cdot s^{-1}\right)$ & $S^{\prime}\left(m^{-1} s^{-1}\right)$ & $w_{e}$ \\
$\begin{array}{c}\text { Standard identification } \\
\text { Coupled identification } \\
\text { (recovery from sample } \\
\text { (a)) }\end{array}$ & $1.2410^{-9}$ & 2.58 & $1.4310^{-7}$ & $2.7210^{-7}$ & $20.1 \%$ \\
\hline
\end{tabular}

359

360 In optimizing $S^{\prime}$ we were able to find a relative good fit of the average moisture content

361 evolution obtained from a dozen weighing measurements and the only two moisture

362 profiles recorded at 7 and 15 days (with gamma measurements once again exhibiting

363 dispersions) with higher moisture content values recorded on the second face. The

364 coupled identification method leads to detecting clearer moisture exchange differences

365 between the two faces, as presented by the moisture profiles in Fig. 7. The standard

366 identification presents two main defaults, less in accordance with the experimental

367 profiles: it quickly leads to high moisture content to both faces and in opposite the

368 moisture content in the middle of the sample should remain low. At last, by considering

369 the only weighing, it cannot easily estimate separated values for the two surface exchange

370 coefficients. 




372 Fig. 7 Results from standard and coupled (recovery from sample (a)) identification, in terms of moisture content profiles for sample (b)

374 The two profiles seem to be sufficient for determining the nonlinear characteristic of the 375 diffusion process, as observed by comparing diffusion coefficient variations vs. the 376 moisture content (Fig. 8) derived by both the standard and coupled identifications.

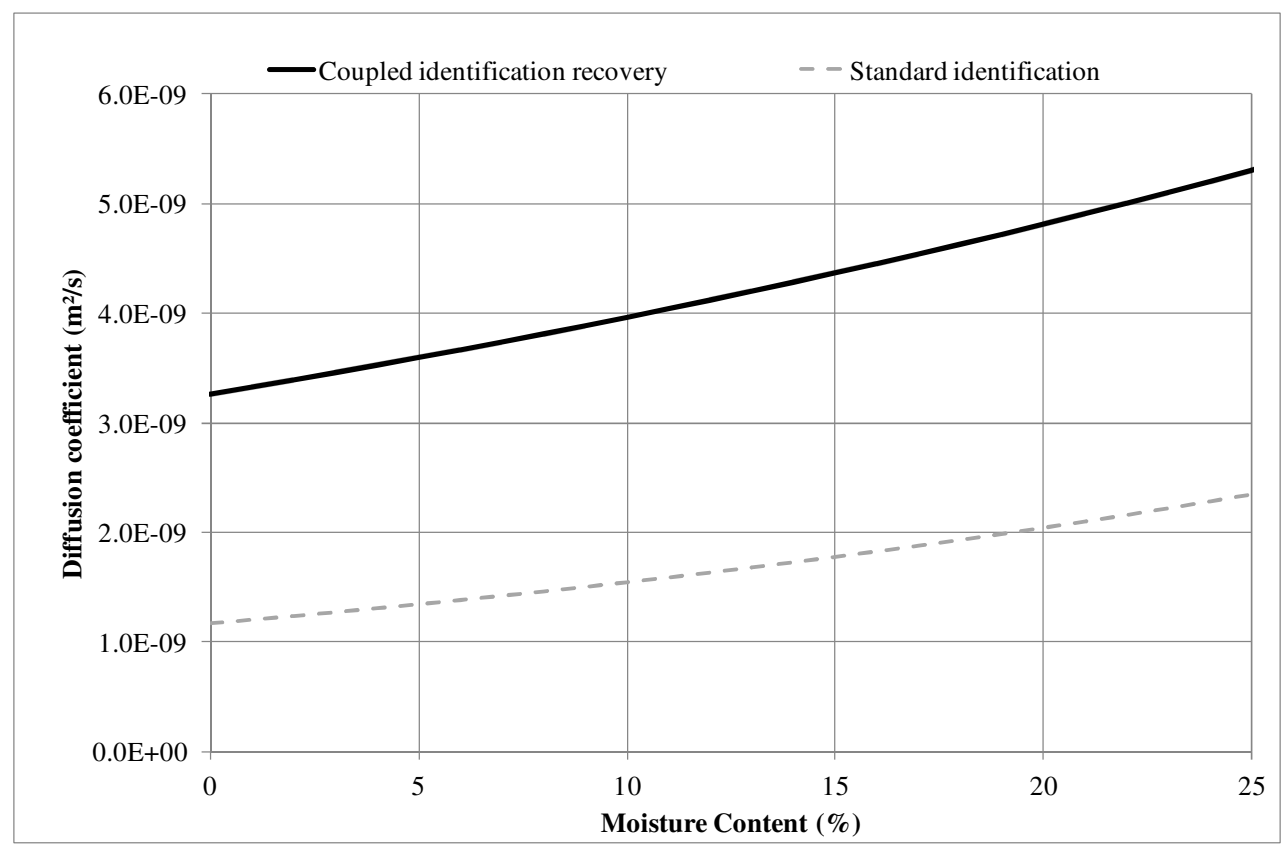

378 Fig. 8 Diffusion coefficient vs. moisture content within the hygroscopic domain, as provided by the identification methods performed on the results of sample (b) 
380 As a matter of fact, this second experiment confirms the differences between coupled and 381 standard identification techniques, with the latter most likely yielding less intrinsic 382 results. In this case, the numerical profile with the coupled method allows for a better 383 agreement with the experimental profile.

\section{3 - Diffusion parameters identification for sample (c): scale effect}

385 This experiment on sample (c) is based on the same single-face exposure adopted for 386 sample (a). As specified in Figure 1, the dimensions and moisture conditions of sample 387 (c) are different in that sample (c) has been previously conditioned in a dry chamber 388 corresponding to the equilibrium moisture content around 9.5\%. In terms of average moisture, the evolution of this sample is plotted in Fig. 9.

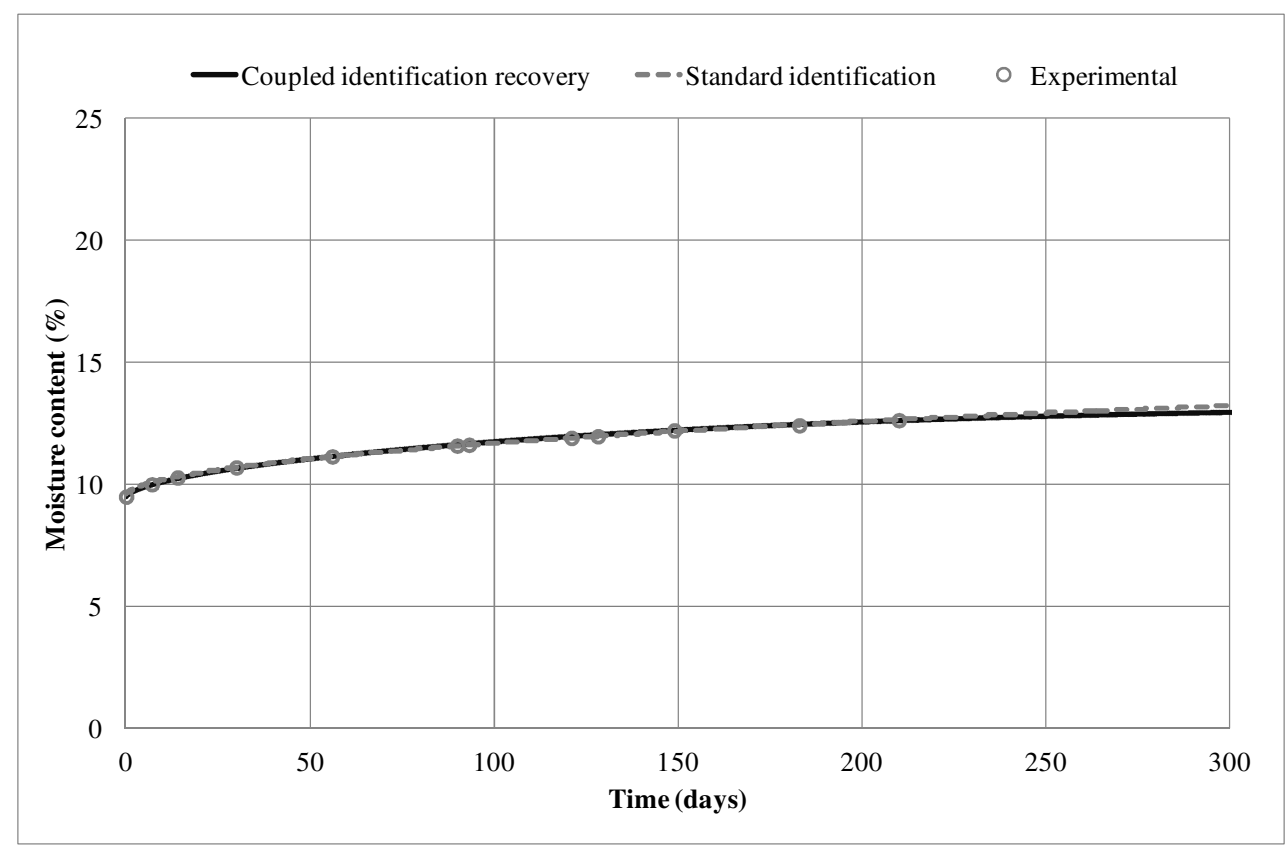

391 Fig. 9 Results of the coupled identification method with recovery from sample (a) and 392 standard identification, in terms of average moisture content evolution for sample (c)

393 The experimental test was stopped after 220 days and a dozen weighing measurements,

394 with the average water content equaling about 13\%. At equilibrium, the moisture content might have been about $15 \%$ according to Loulou (2013). The three profiles measured at 
397 identification methods are summarized in Tab 3.

Tab 3 Diffusion parameters for sample (c)

\begin{tabular}{ccccc}
\hline Identification method & $D_{o}\left(m^{2} \cdot s^{-1}\right)$ & $k$ & $S\left(m \cdot s^{-1}\right)$ & $w_{e}$ \\
Standard identification & $9.33 .10^{-10}$ & 4.64 & $2.64 .10^{-6}$ & $14.8 \%$ \\
$\begin{array}{c}\text { Coupled identification } \\
\text { (recovery from sample (a)) }\end{array}$ & $\mathbf{3 . 2 7 . 1 0 ^ { - 9 }}$ & $\mathbf{1 . 9 4}$ & $2.3510^{-7}$ & $13.3 \%$ \\
\hline
\end{tabular}

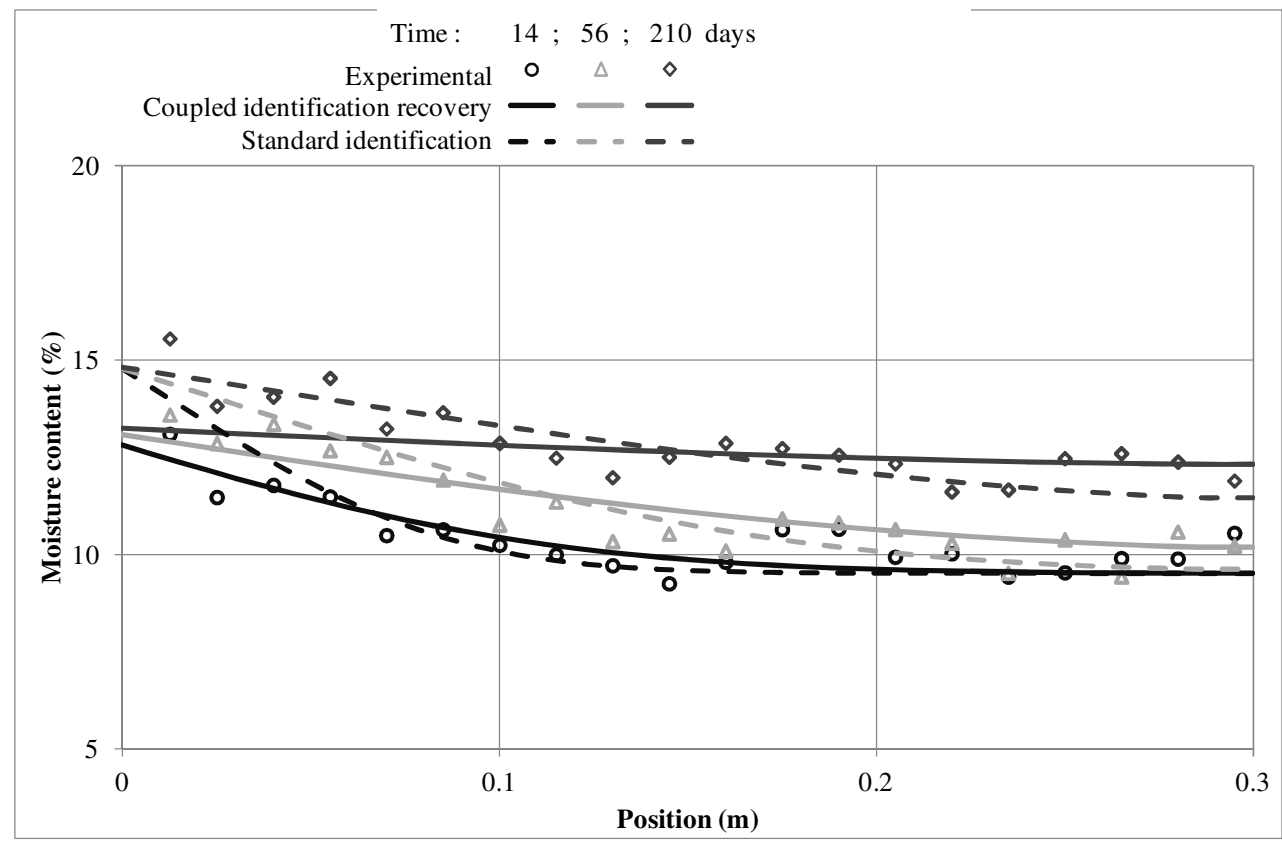

Fig. 10 Results from the standard and coupled (with recovery from sample (a)) identification, in terms of moisture content profiles for sample (c)

403 Lastly, this third experiment again confirms the differences between coupled and standard 404 identification as the numerical profiles with the coupled method allows, in this last case, 405 for a better correlation with the experimental profiles: the moisture content evolution 406 estimated on the exposed face is better predicted in the first moments (days 14 and 56), 407 as the moisture content distribution in the main part (from position 0.1 to $0.3 \mathrm{~m}$ ) of the 408 sample (until day 210). We can just note that the equilibrium moisture content seems to 409 be a little weak regarding to the value recorded near the exposed face, this time better 
410 represented by standard identification's last profile (days 210). More specifically, the two

411 methods differ in terms of hydrous convection kinetics near the exchange surface.

412 Significant differences are also observed in terms of diffusion coefficient evolution as a

413 function of water content (Fig. 11).

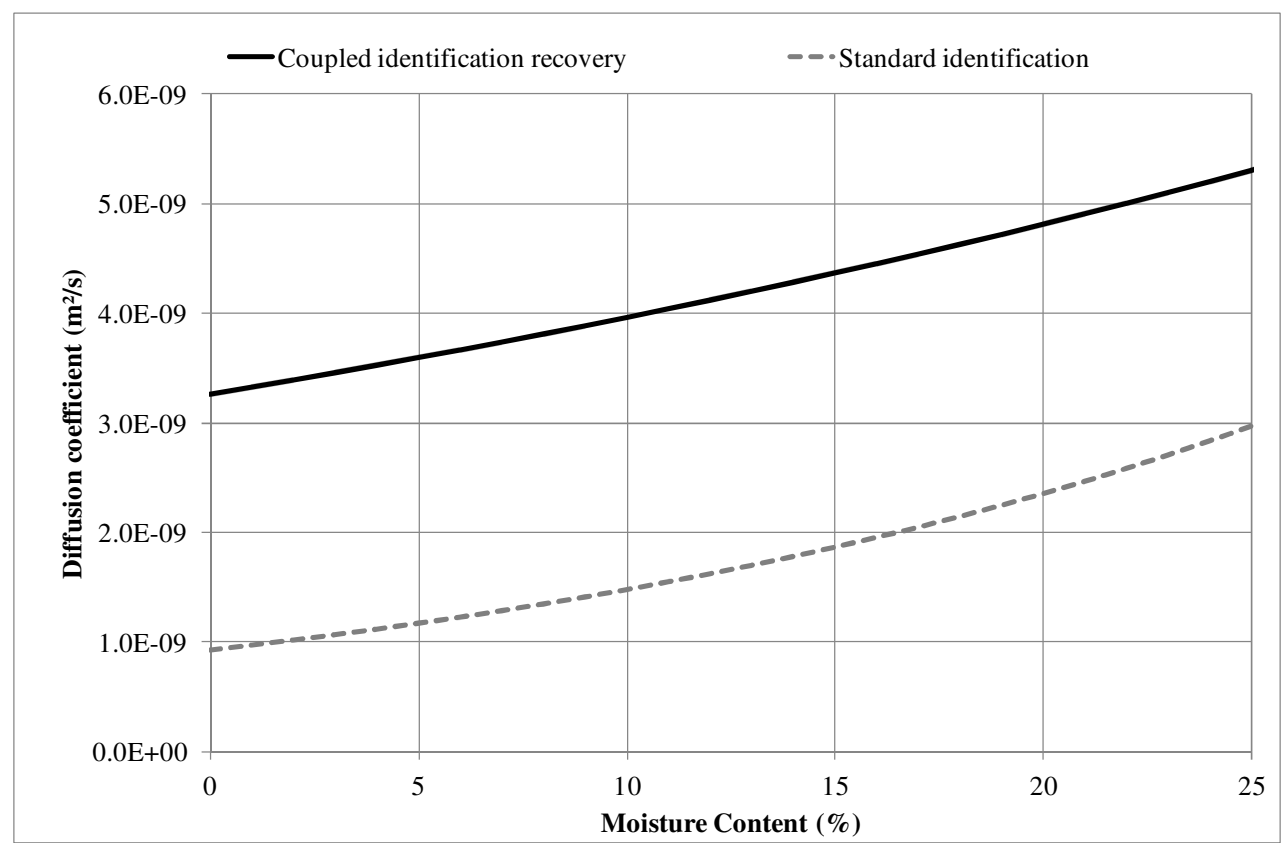

Fig. 11 Diffusion coefficient vs. moisture content within the hygroscopic domain, as output by the two identification methods performed on the results of sample (c)

\section{4 - Discussion}

418 The difference between these two identification methods (standard and coupled), as

419 compared for sample (a), may be explained in a series of remarks. First of all, because 420 the standard identification is based on a global, or averaged, experimental moisture 421 content monitoring, the number of diffusion parameters is capable of inducing selfoptimization, in correlation with one measurement point per time interval. In this case,

423 the surface exchange coefficient $S$ can correct an error when characterizing the diffusion 424 coefficient determination; moreover, as shown in Fig. 3, various identified parameters 425 may be consistent with the time - average moisture content curve. Hence, the standard 426 identifications for samples (a), (b) and (c) show significant differences on diffusion 
coefficients (Fig. 12). The moisture content profiles given by gammadensimetry method

428 make it possible to correct the specimen diffusion parameters along the moisture content

429 gradient. All experimental time points represent a numerical constraint for the inverse

430 method algorithm, thus facilitating the optimization of diffusion parameters. The unique

431 couple of diffusion coefficients identified in the reference case (i.e. sample (a)), along

432 with the proposed coupled method, is able to reproduce both average moisture content

433 evolution and profiles for the two other study cases with different boundary conditions

434 and geometric dimensions (samples (b) and (c)).

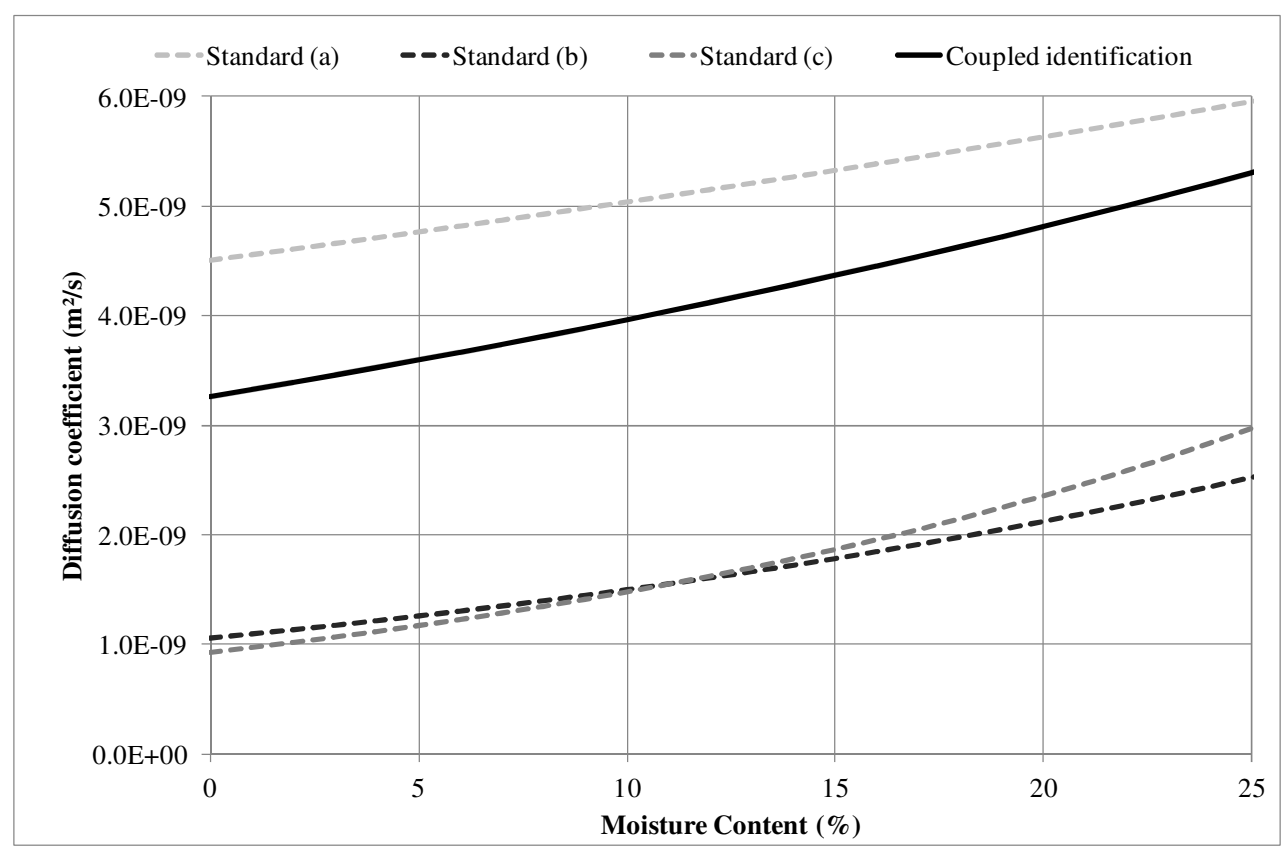

Fig. 12 Diffusion coefficient vs. moisture content within the hygroscopic domain, as provided by the two identification methods performed on the results of samples (a), (b) and (c)

439 Our proposed experimental method deals with robustness of identification of diffusion 440 parameters, usually made with only weighing: with a spatial measurement of water 441 content distribution we fill in a lack of information that could fault any sophisticated 442 model. Indeed, this study has exposed the remediable flaws of the standard identification 443 method, the weighing appearing insufficient with sparsely measurement points far from 
equilibrium. Although characterization does allow for an acceptable modeling of the

445 average moisture content evolution, it is not always possible to easily reproduce the

446 profiles within the material. The balance between the hydrous exchange by convection

447 and diffusion in the material can lead to a significant number of satisfactory datasets for

448 the average moisture content. Moreover, should the experimental time frame prevent

449 achieving moisture equilibrium, the diffusion kinetics errors can lead to an incorrect

450 extrapolation of equilibrium moisture and induce errors in the estimation of sorption

451 isotherms. In this case, a better characterization of diffusion properties, and more

452 specifically convective parameters, at the beginning of the test can increase diffusion

453 extrapolation accuracy. The moisture content profiles do yield better information for a

454 better fit of the time moisture content evolution.

455 Although these profiles contain a high level of experimental noise, the coupled 456 characterization method does enable constraining the minimization algorithm in order to 457 limit the number of possible combinations, in terms of diffusion properties, by 458 highlighting the moisture effects due to convective processes. Such a feature makes this 459 method more accurate and robust with sets of parameters intrinsic to the studied material, 460 thus offering, via the use of a diffusion model, a more realistic prognosis beyond 461 experimental observations. At last, it may be useful for applications in the field of in-situ 462 monitoring of structural timber elements and it will certainly be improved in the future 463 thanks to more elaborated models.

\section{4 - Conclusion and outlook}

465 This work has proposed an experimental protocol for characterizing nonlinear diffusion 466 properties. The coupling between a simple weighing of samples, corresponding to an 467 average moisture variation identification, with a gammadensimetry technique has been 
presented. Such a coupling allows for a realistic separation between diffusion kinetics

469 through the cross-section and the moisture content surface exchange. Moreover, the

470 nonlinear diffusion coefficient is better determined by taking into account the moisture

471 content profiles given by the gamma-ray method. Scale effects, as well as the studied

472 boundary and initial conditions, enable concluding the robustness of this approach in

473 measuring some really intrinsic properties.

474 Moisture profiles have provided us with certain information during the start of adsorption;

475 they allow for a better identification of the initial diffusion times in the vicinity of

476 boundary elements. This additional information increases the model's potential to predict

477 moisture content evolution vs. time. We are now able to propose other experimental 478 protocols for characterizing diffusion without finding moisture equilibrium. In this 479 specific case, this algorithm may be used to determine diffusion properties during 480 moisture buffer tests in which outdoor humidity conditions are in a harmonic solicitation 481 pattern.

482 Regarding the monitoring of timber structures, the diffusion behavior of elements must 483 be known in order to predict long-term mechanical responses and durability of the 484 structures. The gammadensimetry technique offers certain information about the moisture 485 content gradient state; however, this method cannot be generalized to outdoor monitoring. 486 A perspective on this work is the development of a non-destructive method to allow 487 measuring moisture gradients in the cross-section. One solution consists of replacing the 488 gammadensimetry method by a $2 \mathrm{D}$ or $3 \mathrm{D}$ resistive method coupled with a multiplexing 489 technology and a numerical inversion method. 
491 Baettig R., Rémond R., Perré P., Measuring moisture content profiles in a board during drying: a polychromatic x-ray system interfaced with a vacuum/pressure laboratory kiln, Wood Science and Technology, 40(4), 2006, pp. 261-274.

494 Colmars J., Dubois F., Gril J., One-dimensional discrete formulation of a hygrolock 495 model for wood hygromechanics, Mechanics of Time-Dependent Materials, 18(1), 2014, 496 pp. 309-328.

497 Da Rocha M.C., Da Silva L.M., Appoloni C.R., Portezan Filho O., Lopes, F., Melquiades, 498 F.L., et al., Moisture profile measurements of concrete samples in vertical water flow by 499 gamma ray transmission method,. Radiat Phys Chem, 61 (3-6), 2001, pp.567-9.

500 Dubois F., Petit C., Sauvat N., Peuchot B., Diagnostic et comportement des ponts à 501 ossature bois : Application au pont de Merle, European Journal of Civil Engineering, 10 502 (2), 2006, pp. 191-208

503 Dubois F., Husson J.M., Sauvat N., Manfoumbi N., Modeling of the viscoelastic 504 mechano-sorptive behavior in wood, Mechanics of Time-Dependent Materials, 16(4), 505 2012, pp. 439-460.

506 Droin Josserand A., Vergnaud J.M. Taverdet, J.L. Modeling the process of moisture 507 absorption in three dimensions by wood samples of various shapes: cubic, 508 parallelepipedic. Wood Science and Technology, 17. VIII., Vol 23, Issue 3, 1989, pp.259509271.

510 Ferraz E.S.B., Aguiar O., Gamma-ray attenuation technique for determining density and 511 water content of wood samples, IPEF, 30, ago.1985, pp 9-12.

512 Frandsen H.L., Damkilde L., Svensson S., A revised multi-Fickian moisture transport 513 model to describe non-Fickian effects in wood, Holzforschung, 61, 2007, pp. 563-572. 
514 Jakiela S., Bratasz L., Kozlowski R., Numerical modeling of moisture movement and

515 related stress filed in lime wood subjectd to changing climate conditions, Wood Science 516 and Technology, 42(21), 2008, pp. 21-37.

517 Kelley C.T., Iterative Methods for Optimization, North Carolina State University.

518 Raleigh, Society for Industrial and Applied Mathematics, 1999, pp. 135-141.

519 Kouchade A.C., Détermination en routine de la diffusivité massique dans le bois par

520 méthode inverse à partir de la mesure électrique en régime transitoire, $\mathrm{PhD}$ Thesis, 521 Engref, Nancy, 2004.

522 Krabbenhoft K., Moisture transport in wood: a study of physical-mathematical models

523 and their numerical implementation. PhD Thesis, Department of Civil Engineering

524 Technical University of Denmark, 2003.

525 Krabbenhoft K., Damkilde L., A model for non Fickian moisture transfert in wood, 526 Materials and Structures, 37, 2004, pp. 615-622.

527 Lagarias J.C., Reeds J. A., Wright M. H., Wright P. E., Convergence Properties of the 528 Nelder-Mead Simplex Method in Low Dimensions, SIAM Journal, 1998, pp. 112-147.

529 Lasserre B., Modélisation thermo-hygro-mécanique du comportement différé de poutres

530 de structure en bois, $\mathrm{PhD}$ Thesis, University of Bordeaux I, 2000.

531 Liu J.Y., Simpson W.T., Inverse determination of diffusion coefficient for moisture 532 diffusion in wood. Proceedings of 33rd ASME National Heat Transfer Conference : Heat 533 and mass transfer in porous media, August 15-17, 1999, Albuquerque, New Mexico.

534 Loulou L., Durabilité de l'assemblage mixte bois-béton collé sous chargement hydrique, 535 PhD Thesis, Université Paris Est, 2013.

536 Manfoumbi N., Adaptation réglementaire de l'Eurocode 5 aux essences tropicales dans 537 un climat tropical, PhD Thesis, University of Limoges, 2012. 
538 Manfoumbi N., N'Guyen T. A., Angellier N., Dubois F., Ulmet L., Sauvat N.,

539 Experimental and numerical aspects in diffusion process characterization in tropical 540 species, European Journal of Environmental and Civil Engineering, 18 (9), 2014, pp. $541 \quad 963-982$.

542 Merakeb S., Dubois F., Petit C., Sauvat N., Couplage hydromécanique dans le processus 543 de diffusion dans le bois, European Journal of Civil Engineering, 10 (2), 2006, pp. 225544251

545 Merakeb S., Dubois F., Petit C., Modeling of the sorption hysteresis for wood, Wood 546 Science and Technology, 43 (7-8), 2009, pp. 575-589.

547 Nedler J.A., Downhill simplex method, Computer Journal, 7, 1965, pp. 308-313.

548 Olek W., Perre P., Weres J., Implementation of a relaxation equilibrium term in the 549 convective boundary condition for a better representation of the transient bound water 550 diffusion in wood, Wood Science and Technology, 45, 2011, pp. 677-691.

551 Olek W., Rémond R., Weres J., Perré P., Non-Fickian moisture diffusion in thermally 552 modified beech wood analyzed by the inverse method, International Journal of Thermal 553 Sciences, 109, 2016, pp. 291-298.

554 Da Silva W.P., Da Silva L.D., Silva E., C.D.M.P.S., Nascimento P.L., Optimization and 555 simulation of drying processes using diffusion models : application to wood drying using 556 forced air at low temperature, Wood Science and Technology, 45, 2011, pp.787-800.

557 Perre P., Degiovanni A., Simulation par volumes finis des transferts couplés en milieux 558 poreux anisotrope : séchage du bois à basse et haute température, International Journal 559 of Heat and Mass Transferts, 33(11), 1990, pp.2463-2478.

560 Perre P., Turner I.W., A mesoscopic drying model applied to the growth rings of 561 softwood: mesh generation and simulation results, Maderas. Cienca y tecnologia, 10(3), 562 2008, pp. 251-274. 
563 Rozas C., Tomaselli I., Zanoelo E. F., Internal mass transfer coefficient during drying of

564 softwood (Pinus elliottii Engelm.) boards, Wood Science and Technology, 43 2009, pp.

$565361-373$.

566 Villain G and Thierry M.. Gammadensimetry: a method to determine drying and 567 carbonation profiles in concrete, $N D \& E$ Int., 39 (4), 2006, pp.328-337.

568 Zhou Q., Cai Y., Xu Y., Zhang X., Determination of moisture diffusion coefficient of 569 larch board with finite difference method, BioRes, 6 (2), 2011, pp. 1196-1203. 